Journal Club

Editor's Note: These short, critical reviews of recent papers in the Journal, written exclusively by graduate students or postdoctoral fellows, are intended to summarize the important findings of the paper and provide additional insight and commentary. For more information on the format and purpose of the Journal Club, please see http://www.jneurosci.org/misc/ifa_features.shtml.

\title{
Insights into the Cellular and Molecular Contributions of MeCP2 Overexpression to Disease Pathophysiology
}

\author{
M. Morgan Taylor* and Shachee Doshi* \\ Neuroscience Graduate Group, Perelman School of Medicine, University of Pennsylvania, Philadelphia, Pennsylvania 19104 \\ Review of Dastidar et al. and $\mathrm{Na}$ et al.
}

Rett syndrome (RTT) is a devastating and currently incurable neurodevelopmental disease that primarily affects females. RTT is caused by loss of the X-linked gene methyl$C p G$-binding protein 2 (MECP2). The resulting decreases in MeCP2 protein levels lead to symptoms such as developmental regression, intellectual and learning difficulties, stereotyped hand motions, impaired motor skills, loss of language, ataxia, and seizures (Ramocki and Zoghbi, 2008). While there is evidence to suggest that the primary role of $\mathrm{MeCP} 2$ is transcriptional repression, it has also been shown to selectively enhance gene expression (Chahrour et al., 2008), and its specific roles in neuronal function remain controversial.

Whereas much effort has been devoted to understanding the mechanisms of RTT pathology, relatively little is known regarding $M E C P 2$ duplication syndrome (MECP2 DS). In contrast to RTT, MECP2 DS affects mostly males and is caused by duplications of the MECP2 gene, which increases MeCP2 protein levels. MECP2 DS patients exhibit symptoms that, in some cases, resemble those seen in RTT, including mental retar-

Received April 27, 2012; revised May 21, 2012; accepted May 22, 2012.

This work was supported by the Neuroscience Graduate Group/Biomedical Graduate Studies (Perelman School of Medicine, University of Pennsylvania) and the Systems and Integrative Biology Training Grant (T32GM07517). We are extremely grateful to the mentorship of Dr. Todd Lamitina, Dr. Doug Coulter, and Dr. Greg Carlson.

${ }^{*}$ M.M.T. and S.D. contributed equally to this work.

Correspondence should be addressed to M. Morgan Taylor, University of Pennsylvania, 3620 Hamilton Walk, 140 John Morgan Building, Philadelphia, PA 19104-6074. E-mail: taym@mail.med.upenn.edu.

DOI:10.1523/JNEUROSCI.2043-12.2012

Copyright $\odot 2012$ the authors $\quad 0270-6474 / 12 / 329451-03 \$ 15.00 / 0$ dation, impaired development of speech and motor control, and seizures (Ramocki and Zoghbi, 2008). However, recent work suggests that neurodegeneration may play a role in the pathology of MECP2 DS patients (Reardon et al., 2010), whereas neurodegeneration has not thus far been associated with RTT. Two recent articles in The Journal of Neuroscience provide insight into MECP2 DS by characterizing animal and cell models of MeCP2 overexpression. Based on results from these studies, we present a hypothesis to link enhanced MeCP2 function to both synaptic deficits and neurodegeneration.

In the first study, Na et al. (2012) characterized a mouse model of MECP2 DS. Neuronspecific $\mathrm{MeCP} 2$ overexpression gave rise to animals with decreased body weight, deficits in motor coordination, increased anxiety-like behavior, and impaired learning and memory, similar to symptoms observed in human MECP2 DS patients. Electrophysiological recordings in hippocampal brain slices from these mice revealed decreases in long-term potentiation (LTP) and the probability of neurotransmitter release, indicative of attenuated synapse strength and activity. Remarkably, administration of a histone deacetylase (HDAC) inhibitor reversed defects in miniature EPSCs observed in cultured hippocampal neurons, suggesting that HDAC-dependent transcriptional repression mediates synaptic defects associated with MeCP2 overexpression. Unexpectedly, the authors found that one previously characterized MeCP2 target gene, brain-derived neurotrophic factor, was not upregulated in their model, suggesting that MeCP2- dependent transcription of other genes must mediate the observed synaptic defects.

In the second article, Dastidar et al. (2012) studied the roles of two previously identified MeCP2 splice variants, MeCP2-e1 and MeCP2-e2, in cultured mouse cerebellar neurons (CCNs). The authors demonstrated that MeCP2-e1 and MeCP2-e2 exhibit different expression profiles and play divergent functional roles in CCNs. When cells were subjected to apoptosis-inducing conditions, mRNA expression of the e 2 isoform was upregulated and expression of the e1 isoform was downregulated. Overexpression of MeCP2-e2 promoted apoptosis in healthy cells, whereas MeCP2-e2 knockdown rendered cells resistant to the apoptosis-inducing treatment. In contrast, neither overexpression nor knockdown of MeCP2-el affected cell viability. This study further showed that FoxG1, a transcription factor whose dysregulation has also been implicated in RTT, preferentially interacts with MeCP2-e2 and that this interaction restricts the neurotoxic effects of MeCP2-e2. The authors concluded that MeCP2-e2 promotes neuronal cell death when unchecked by FoxG1. This paper provides the first evidence of a functional difference between $\mathrm{MeCP} 2$ isoforms, highlighting the necessity of investigating differences between protein isoforms in disease pathogenesis.

It is important to note that the apoptosispromoting isoform of $\mathrm{MeCP} 2, \mathrm{MeCP} 2-\mathrm{e} 2$, was the one overexpressed in neurons in the $\mathrm{Na}$ et al. (2012) mouse model. Both papers therefore characterized increased expression of MeCP2-e2. Na et al. (2012) examined the 
effects of MeCP2-e2 overexpression primarily in vivo in the context of modeling symptoms of MECP2 DS, whereas Dastidar et al. (2012) explored the role of $\mathrm{MeCP} 2$ protein by examining effects of specific isoform overexpression in cerebellar neurons in vitro. In the animal model, the primary nonbehavioral phenotype associated with overexpression was altered synaptic activity in an intact network, and $\mathrm{Na}$ et al. (2012) did not explicitly look at cell viability, which was the main defect seen in cultured neurons in Dastidar et al. (2012).

The relationship between synaptic defects and cell death, the phenotypes found in these two studies, remains unclear because the experiments were conducted in different brain tissues and different preparations. It is possible that $\mathrm{MeCP} 2$ function has cell-type and tissue-specific roles, such that its overexpression induces cell death in cerebellum while coordinately altering synaptic function in hippocampus. Indeed, gene expression studies have shown that modulating MeCP2 dosage in mice results in different gene expression changes in different regions of the brain, implying that the protein may play divergent roles in different tissues (BenShachar et al., 2009).

Synaptic deficits and cell death are also characteristic phenotypes of Alzheimer's disease, and evidence suggests that accumulations of misfolded $\mathrm{A} \beta$ oligomers underlie both of these phenotypes (Harmeier et al., 2009). Protein misfolding and aggregation are not commonly cited characteristics of RTT or MECP2 DS, but recent evidence bridging apoptosis and synaptic activity may provide a different unifying mechanism accounting for the findings of both Dastidar et al. (2012) and $\mathrm{Na}$ et al. (2012). Studies have found that expression of the GluA2 subunit of glutamatergic AMPA receptors (AMPARs) is regulated by the repressor element-1 silencing transcription factor (REST), which is normally maintained at low levels of activity in mature neurons. Increased REST activity in neurons occurs under conditions of cellular stress such as ischemia and epilepsy and leads to silencing of GluA2 expression (Noh et al., 2012). Decreased levels of GluA2 have been associated with a decrease in LTP (Romberg et al., 2009), and loss of GluA2 is associated with cell death resulting from excitotoxicity via GluA2-lacking, calcium-permeable AMPARs (Gorter et al., 1997). Intriguingly, $\mathrm{MeCP} 2$ is part of the complex recruited by REST in neurons (Ballas et al., 2005), and increased expression of MeCP2 has been linked to decreased GluA2 subunit expres-

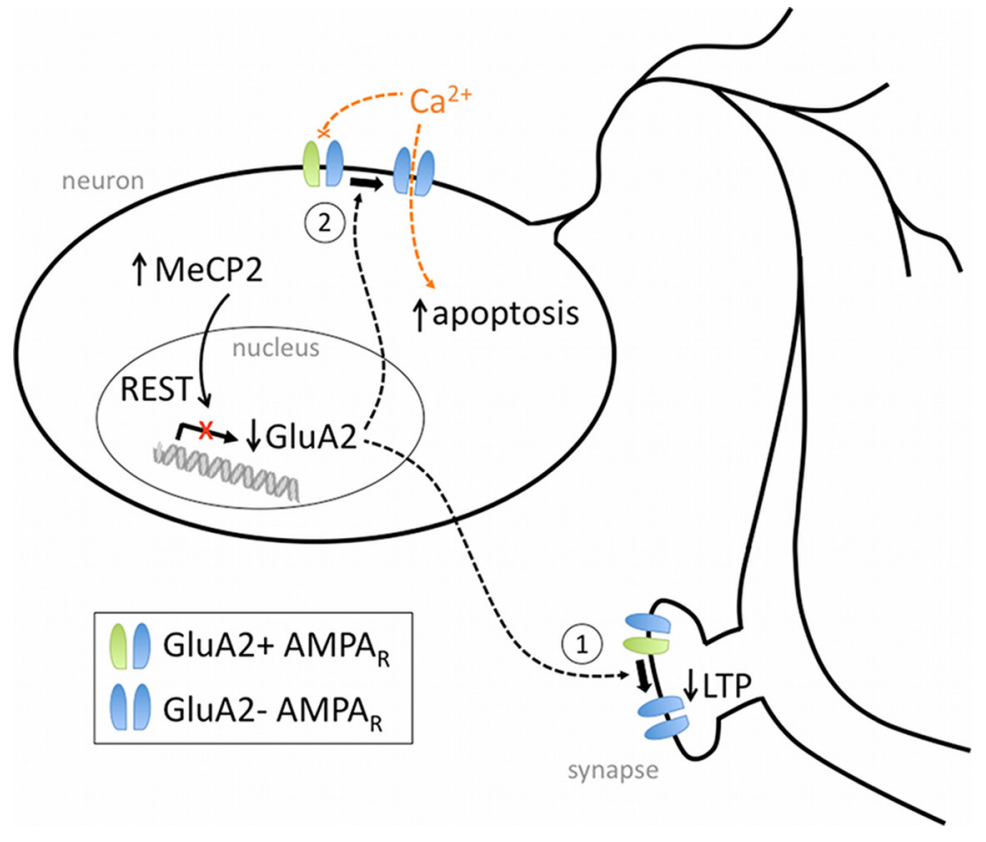

Figure 1. Schematic illustration depicting a proposed effect of MeCP2 overexpression. In this model, MeCP2 participates in the REST pathway to repress gene expression of GluA2 subunits (green). Decreased expression of GluA2 results in an increase in the proportion of AMPA receptors lacking this subunit (indicated here by all-blue receptors), which can lead to deficits in LTP (1) and increased calcium-mediated apoptosis (2).

sion and synaptic changes (Qiu et al., 2012). It is thus tempting to speculate that MeCP2 overexpression acts via REST to affect both synaptic activity and cell death (Fig. 1).

Analysis of REST activation and GluA2 expression in $\mathrm{MeCP} 2$-overexpressing mice $(\mathrm{Na}$ et al., 2012) and cerebellar cultures (Dastidar et al., 2012) could readily be used to test the proposed model. Given that Na et al. (2012) did not find a statistically significant increase in $\mathrm{MeCP} 2$ expression in cerebellar tissue, in which Dastidar et al. (2012) found an apoptotic phenotype, neuronal viability and synaptic activity in cerebella as well as hippocampi of these mice should also be examined to confirm the link between cell culture and in vivo effects of $\mathrm{MeCP} 2$ overexpression. Furthermore, given that FoxG1 overexpression and application of HDAC inhibitors rescued cellular phenotypes in vitro, these manipulations should be applied in vivo to $\mathrm{MeCP} 2$-overexpressing mice to determine whether the behavioral and cellular phenotypes are rescued. Such a rescue would further support a causal link between the cellular and behavioral deficits seen in $\mathrm{MeCP} 2$-overexpressing mice, illuminating promising avenues for therapeutic interventions in MECP2 DS.

Much research has been devoted to understanding and characterizing the effects of decreased expression and loss of function of $\mathrm{MeCP} 2$, particularly as it relates to RTT. The studies by Na et al. (2012) and Dastidar et al. (2012) introduce new perspectives on
MeCP2 function and provide necessary models for MECP2 DS research. As the symptoms of MeCP2-related disorders indicate, understanding disease models and mechanisms associated with MeCP2 overexpression has wider implications beyond the cell for circuit-level and behavioral functions. These two studies represent important new steps toward understanding and eventually curing MeCP2-associated diseases.

\section{References}

Ballas N, Grunseich C, Lu DD, Speh JC, Mandel G (2005) REST and its corepressors mediate plasticity of neuronal gene chromatin throughout neurogenesis. Cell 121:645-657.

Ben-Shachar S, Chahrour M, Thaller C, Shaw CA, Zoghbi HY (2009) Mouse models of MeCP2 disorders share gene expression changes in the cerebellum and hypothalamus. Hum Mol Genet 18:2431-2442.

Chahrour M, Jung SY, Shaw C, Zhou X, Wong ST, Qin J, Zoghbi HY (2008) MeCP2, a key contributor to neurological disease, activates and represses transcription. Science 320:1224-1229.

Dastidar SG, Bardai FH, Ma C, Price V, Rawat V, Verma P, Narayanan V, D'Mello SR (2012) Isoform-specific toxicity of Mecp2 in postmitotic neurons: suppression of neurotoxicity by FoxG1. J Neurosci 32:2846-2855.

Gorter JA, Petrozzino JJ, Aronica EM, Rosenbaum DM, Opitz T, Bennett MV, Connor JA, Zukin RS (1997) Global ischemia induces downregulation of GluR2 mRNA and increases AMPA receptor-mediated $\mathrm{Ca} 2+$ influx in hippocampal CA1 neurons of gerbil. J Neurosci 17:6179-6188. 
Harmeier A, Wozny C, Rost BR, Munter LM, Hua $\mathrm{H}$, Georgiev O, Beyermann M, Hildebrand PW, Weise C, Schaffner W, Schmitz D, Multhaup G (2009) Role of amyloid-beta glycine 33 in oligomerization, toxicity, and neuronal plasticity. J Neurosci 29:7582-7590.

$\mathrm{Na}$ ES, Nelson ED, Adachi M, Autry AE, Mahgoub MA, Kavalali ET, Monteggia LM (2012) A mouse model for MeCP2 duplication syndrome: MeCP2 overexpression impairs learning and memory and synaptic transmission. J Neurosci 32:3109-3117.

Noh KM, Hwang JY, Follenzi A, Athanasiadou R,
Miyawaki T, Greally JM, Bennett MV, Zukin RS (2012) Repressor element-1 silencing transcription factor (REST)-dependent epigenetic remodeling is critical to ischemiainduced neuronal death. Proc Natl Acad Sci U S A 109:E962-E971.

Qiu Z, Sylwestrak EL, Lieberman DN, Zhang Y, Liu XY, Ghosh A (2012) The Rett syndrome protein $\mathrm{MeCP} 2$ regulates synaptic scaling. J Neurosci 32:989-994.

Ramocki MB, Zoghbi HY (2008) Failure of neuronal homeostasis results in common neuropsychiatric phenotypes. Nature 455:912-918.
Reardon W, Donoghue V, Murphy AM, King MD, Mayne PD, Horn N, Birk Møller L (2010) Progressive cerebellar degenerative changes in the severe mental retardation syndrome caused by duplication of MECP2 and adjacent loci on Xq28. Eur J Pediatr 169: 941-949.

Romberg C, Raffel J, Martin L, Sprengel R, Seeburg PH, Rawlins JN, Bannerman DM, Paulsen O (2009) Induction and expression of GluA1 (GluR-A)-independent LTP in the hippocampus. Eur J Neurosci 29: 1141-1152. 